\title{
EDITORIAL
}

\section{EL MEDICAMENTO COMO SOLUCIÓN Y COMO PROBLEMA PARA LA SALUD PÚBLICA. UNA BREVE INCURSIÓN A LOS OBJETIVOS DE LA FARMACOEPIDEMIOLOGÍA}

\section{Francisco J de Abajo}

División de Farmacoepidemiología y Farmacovigilancia. Agencia Española del Medicamento. Ministerio de Sanidad y Consumo

En el presente número de la Revista Española de Salud Pública aparecen una serie de artículos que tienen al medicamento como elemento común. Por esa razón se me ha pedido un comentario acerca de la importancia del medicamento como determinante de salud $\mathrm{y}$, por tanto, como foco de atención en estudios de salud pública.

Hoy día, los estudios epidemiológicos que tienen su centro de interés en el medicamento se pueden agrupar dentro de una disciplina conocida como farmacoepidemiología. Académicamente se define como la aplicación del razonamiento, métodos y conocimiento epidemiológico al estudio de los usos y los efectos de los medicamentos en las poblaciones humanas ${ }^{1}$ y surge de la fusión de la Farmacología Clínica (que presta el contenido: el uso de medicamentos en el ser humano) con la Epidemiología (que presta su método). Puede decirse que la farmacoepidemiología adquiere cuerpo de doctrina en la década de los ochenta cuando se publican varios libros que hoy son una referencia obligada ${ }^{1-3}$. Pero el inicio práctico de la misma es, sin duda, anterior. Hay que retrotraerse al menos hasta la década de los sesenta. Es entonces cuando el desastre de la

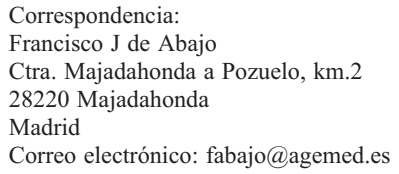

talidomida fija en nuestra conciencia, como un imperativo ético y científico, la necesidad de estudiar los efectos adversos de los medicamentos aún después de comercializados, dando inicio a los programas de farmacovigilancia, y es también entonces cuando se comienzan a realizar los primeros estudios de utilización de medicamentos, que muestran la diversidad en los patrones de uso, y se analizan sus causas y sus consecuencias ${ }^{4}$. El surgimiento del interés en esta época por el estudio del medicamento a escala social no es casualidad, desde luego. Es fruto de la industrialización progresiva del medicamento que dio lugar, por un lado, a la revolución terapéutica posterior a la segunda guerra mundial y, por otro, al acceso generalizado de la población a este bien de consumo tan particular. En 30 años se pasó de tener apenas una decena de medicamentos activos y fiables a disponer de un número considerable de ellos para una variedad importante de enfermedades ${ }^{5} \mathrm{y}$, por tanto, de una exposición limitada a una exposición masiva. Fue entonces cuando nos dimos cuenta del impacto del medicamento en la salud pública y de forma natural nació la epidemiología del medicamento.

Es evidente: el medicamento constituye un importante determinante de la salud de los ciudadanos, aunque solo fuera porque de ellos depende en buena medida la capacidad que hoy tiene la Medicina de interrumpir o modificar el curso natural de las enfermedades, de prevenirlas o, en cualquier caso, de 
hacer su peso más liviano. No sorprende, por tanto, que el consumo de medicamentos pueda ser un excelente indicador de la prevalencia de ciertas enfermedades como lo demuestra el estudio de Morant y colaboradores $^{6}$ en el presente número. Pero los medicamentos también pueden eventualmente ser la causa de las enfermedades. Se ha estimado que cerca del $30 \%$ de todas las hospitalizaciones están relacionadas con el uso de medicamentos (enfermedades no tratadas, inadecuada selección del medicamento, reacciones adversas, incumplimiento, interacciones, uso de fármacos sin una indicación apropiada y sobredosis), situando sus costes entre las cinco primeras causas de morbilidad en el mundo desarrollado ${ }^{7}$. Por otro lado, se conoce que aproximadamente el $4 \%$ de las nuevas entidades químicas y biológicas que se introducen en el mercado deben ser retiradas después por el descubrimiento de reacciones adversas no conocidas o no bien cuantificadas durante el desarrollo clínico ${ }^{8}$. Esta proporción estimada a partir de datos del Reino Unido, Estados Unidos y España, durante el período 1974-1993, se ha incrementado con seguridad en la última década $^{9}$. Valgan estas razones para justificar por qué la farmacoepidemiología se ha desarrollado muy fundamentalmente como una herramienta al servicio de la farmacovigilancia, una actividad de salud pública orientada al análisis y gestión de los riesgos de los medicamentos una vez comercializa$\operatorname{dos}^{10}$. Cada vez es más frecuente, no obstante, que los estudios farmacoepidemiológicos se ocupen también de medir la efectividad y la eficiencia de los medicamentos en un contexto clínico real, un conocimiento cada vez más necesario que los ensayos clínicos controlados previos a la comercialización no aportan. Lamentablemente, en muchas ocasiones esta necesidad ha sido utilizada por compañías farmacéuticas para organizar pseudoestudios científicos con un interés eminentemente comercial. En este punto conviene anotar una iniciativa de la Agencia Española del Medicamento para evitar en lo posible este tipo de estrategias y fomentar el uso científico de los llamados genéricamen- te estudios de post-autorización. En junio de 2001 se dio a conocer un informe que estará sometido a comentario y escrutinio público durante 3 o 4 meses y del que se espera salga una iniciativa reguladora que clarifique la situación (para más información consúltese www.agemed.es).

Saber cuánto y cómo se utilizan los medicamentos, e intentar mediante estudios apropiados de intervención modificar su uso irracional, es otro de los grandes objetivos de la farmacoepidemiología. Los estudios de Prieto Yerro y colaboradores ${ }^{11}$ y de Álvarez de Toledo y colaboradores ${ }^{12}$ se pueden inscribir en esta línea, y ambos demuestran la importante labor que farmacólogos clínicos y farmacéuticos pueden y deben desarrollar para contribuir a una mejor calidad técnica y ética del uso de los medicamentos. El estudio de Orden y de la Fuente ${ }^{13}$ cabe inscribirlo también en este área, bien que con la particularidad de que aquí de lo que se trata es del uso de antimicrobianos en animales que, como sugieren los autores, puede ser el origen de graves problemas para la salud pública.

El medicamento, pues, como solución y también como problema en salud pública, y toda una disciplina para estudiar ambos y a la que están llamados a contribuir profesionales de diferentes adscripciones y procedencias, como vemos. Que cunda el ejemplo.

\section{BIBLIOGRAFÍA}

1. Hartzema AG, Porta MS, Tilson HH (eds.). Pharmacoepidemiology: an introduction, 3rd ed, Cincinnati: Harvey Whitney Books; 1998.

2. Strom BL (ed.). Pharmacoepidemiology, 3rd ed, Chichester: John Wiley \& Sons, Ltd; 2000.

3. Laporte JR, Tognoni G. Principios de epidemiología del medicamento. Barcelona: Masson-Salvat (colección Salud Pública); 1993.

4. Einarson TR, Bergman U, Wiholm BE. Principles and practice of pharmacoepidemiology. En: Speight TM, Holford HG, eds, Avery's Drug Treatment, $4^{\text {th }}$ ed, Auckland: Adis International; 1997. 
5. Del Río J. Segunda revolución terapéutica: realidades y proyecciones. En: La nueva frontera del medicamento. Madrid: Instituto de Ciencias del Hombre; 1985.

6. Morant Ginestar C, Criado-Alvarez JJ, García-Pina R y Pérez Garrido B. Estimación de la prevalencia de hipotiroidismo en España a partir del consumo de hormonas tiroideas (1996-1999). Rev Esp Salud Pública 2001; 75:337-344.

7. Johnson JA, Bootman JL. Drug-related morbidity and mortality: a cost-of-illness model. Arch Intern Med 1995, 155:1949-1956.

8. Bakke OM, Manocchia M. De Abajo FJ, Kaitin K. Lasagna L. Drug safety disconitunations in the United Kingdom, the United States and Spain from 1974 through 1993: a regulatory perspective. Clin Pharmacol Ther 1995; 58:108-117.

9. Arnáiz JA, Carné X, Riba N, Codina C, Ribas J, Trilla A. The use of evidence in pharmacovigilance- case reports as the reference source for drug withdrawals. Eur J Clin Pharmacol 2001; 57: 89-91.
10. De Abajo FJ, Montero D, Madurga M, Palop R. Análisis y gestión de riesgos en farmacovigilancia. Organización de la farmacovigilancia en España. En: García AG, Gandía L, eds, El ensayo clínico en España. Madrid: Farmaindustria (serie científica); 2001

11. Prieto Yerro C, Laredo Velasco L, Rodríguez Bernardino A, Vargas Castrillón E, Ambit Ávila MI y Merchante Medina A. Modificación en el patrón de uso de Anfotericina B no convencional en el Hospital Clínico San Carlos de Madrid tras una intervención formativa. Rev Esp Salu Pública 2001; 75:353-360.

12. F Álvarez de Toledo A, Arcos González P, Eyaralar Riera T, Abal Ferrer F, A Dago Martínez, Cabiedes Miragaya L et al. Atención farmacéutica en personas que han sufrido episodios coronarios agudos (Estudio TOMCOR). Rev Esp Salu Pública 2001; 75:375-388.

13. Orden Gutiérrez JA y de la Fuente López R. Repercusiones en la salud pública de la resistencia a quinolonas en bacterias de origen animal. Rev Esp Salu Pública 2001; 75:313-320 